\title{
Abundances of Molecular Species in Barnard 68
}

\author{
James Di Francesco ${ }^{1}$, Michiel R. Hogerheijde ${ }^{2}$, William J. Welch \\ Radio Astronomy Laboratory, 601 Campbell Hall, \\ University of California, Berkeley, Berkeley, CA, \\ 94705-3411 U.S.A. \\ Edwin A. Bergin \\ Harvard-Smithsonian Center for Astrophysics \\ 60 Garden Street, MS 42, Cambridge, MA 02138, U.S.A.
}

Received __; accepted _

\footnotetext{
${ }^{1}$ currently at Herzberg Institute of Astrophysics, National Research Council of Canada, 5071 West Saanich Road, Victoria, BC V9E 2E7 Canada

${ }^{2}$ currently at Steward Observatory, University of Arizona, 933 North Cherry Avenue, Tucson, AZ 85721-0065, U.S.A.
} 


\begin{abstract}
Abundances for 5 molecules $\left(\mathrm{C}^{18} \mathrm{O}, \mathrm{CS}, \mathrm{NH}_{3}, \mathrm{H}_{2} \mathrm{CO}\right.$, and $\left.\mathrm{C}_{3} \mathrm{H}_{2}\right)$ and 1 molecular ion $\left(\mathrm{N}_{2} \mathrm{H}^{+}\right)$and upper limits for the abundances of 1 molecule $\left({ }^{13} \mathrm{CO}\right)$ and 1 molecular ion $\left(\mathrm{HCO}^{+}\right)$are derived for gas within the Bok globule Barnard 68 (B68). The abundances were determined using our own BIMA millimeter interferometer data and single-dish data gathered from the literature, in conjunction with a Monte Carlo radiative transfer model. Since B68 is the only starless core to have its density structure strongly constrained via extinction mapping, a major uncertainty has been removed from these determinations. All abundances for B68 are lower than those derived for translucent and cold dense clouds, but perhaps only significantly for $\mathrm{N}_{2} \mathrm{H}^{+}, \mathrm{NH}_{3}$, and $\mathrm{C}_{3} \mathrm{H}_{2}$. Depletion of CS toward the extinction peak of B68 is hinted at by the large offset between the extinction peak and the position of maximum CS line brightness. Abundances derived here for $\mathrm{C}^{18} \mathrm{O}$ and $\mathrm{N}_{2} \mathrm{H}^{+}$are consistent with other, recently determined values at positions observed in common.
\end{abstract}

Subject headings: ISM: molecules — ISM: abundances — ISM: globules — ISM: individual (B68) — stars: formation

\title{
1. Introduction
}

Stars form out of cores of dense molecular gas. Such cores can be embedded deeply within molecular clouds, but numerous examples of isolated cores, e.g., Bok globules (Bok \& Reilly 1947; Clemens \& Barvainis 1988), have been also identified as dark patches of high visual extinction against rich stellar backgrounds. As isolated cores, Bok globules can be relatively free from the influences of nearby star-forming events, and so can provide very 
simple and pristine examples of the physical and chemical structures of cores prior to the formation of low-mass stars. In this paper, we examine the abundances of several species of molecules or molecular ions in the globule Barnard 68 (B68; Barnard 1919).

B68 (also known as CB82 or LDN57) is an Ophiuchus dark cloud, located at $\sim 125 \mathrm{pc}$ (de Geus, de Zeeuw, \& Lub 1989)3, within the Loop I superbubble. Figure 1 shows an $R$-band image of B68 obtained from the CDS/Aladin sky atlas, revealing its compact, roundish morphology. B68 has no evidence of star formation within its interior, e.g., from detections of outflow wings in lines of ${ }^{12} \mathrm{CO}$ (Avery et al. 1987) or from an IRAS point source (Parker 1988) ${ }^{4}$. Furthermore, continuum emission at $\lambda=1.3 \mathrm{~mm}$ toward B68 has not been detected (Reipurth, Nyman, \& Chini 1996; Launhardt \& Henning 1997).

B68 is unique in that it is the only starless Bok globule to have its density structure well defined. Using the extinction of the background Galactic bulge K-giant population, Alves, Lada, \& Lada (2001; ALL01) found an azimuthally-averaged radial column density profile for B68 that was well matched with that of an isothermal, self-gravitating Bonnor-Ebert sphere, specifically one with a near-critical center-to-edge density contrast of 16.5 (Bonnor 1956; Ebert 1955). The robust definition of density within B68 removes a major uncertainty towards determining its molecular abundances. Recently, Bergin et al. (2002) used single-dish maps of B68 in conjunction with models specifically incorporating the ALL01 Bonnor-Ebert density structure to ascertain radial variations in $\mathrm{C}^{18} \mathrm{O}$ and $\mathrm{N}_{2} \mathrm{H}^{+}$abundance, with the lowest values occurring at the highest extinctions within B68. Hotzel et al. (2002)

\footnotetext{
${ }^{3} \mathrm{~A}$ distance range of 60-205 pc for the Ophiuchus dark clouds was formally estimated by de Geus et al. with $125 \mathrm{pc} \pm 25 \mathrm{pc}$ defined as their center.

${ }^{4}$ The $24^{\prime \prime} \times 5^{\prime \prime}\left(\right.$ P.A. of $\left.94^{\circ}\right)$ error ellipse of the IRAS point source 17194-2351, associated with $\mathrm{B} 68$ by Clemens \& Barvainis, is centered $\sim 3^{\prime}$ beyond the opaque edge of B68 seen in Figure 1.
} 
also found evidence for depletion of CO isotopes with extinction from their own single-dish maps and a re-analysis of the ALL01 data. Each group speculated these molecules sublimate from the gas phase onto dust grains at the high densities and low temperatures in the B68 interior. A similar interpretation was made by Tafalla et al. (2002) to explain the radial depletion of $\mathrm{CS}$ and $\mathrm{C}^{18} \mathrm{O}$ in the interiors of 5 other starless cores, although $\mathrm{N}_{2} \mathrm{H}^{+}$was not depleted and $\mathrm{NH}_{3}$ was enhanced at these locations.

We explore further the abundances of several molecules or molecular ions within B68, incorporating the ALL01 Bonnor-Ebert density structure into our own models. New observations of B68 made with the BIMA millimeter array are used to obtain upper limits on abundances of ${ }^{13} \mathrm{CO}$ and $\mathrm{HCO}^{+}$near the extinction peak found by ALL01, i.e., at R.A./decl. $(2000)=17^{h} 22^{m} 38.6^{s},-23^{\circ} 49^{\prime} 46.0^{\prime \prime}$. Upper limits to $\mathrm{C}^{18} \mathrm{O}$ and $\mathrm{N}_{2} \mathrm{H}^{+}$ abundances are also derived at this position, consistent with values derived by Bergin et al. In addition, single-dish detections of lines reported in the literature are used to derive abundance values for $\mathrm{C}^{18} \mathrm{O}, \mathrm{CS}, \mathrm{N}_{2} \mathrm{H}^{+}, \mathrm{NH}_{3}, \mathrm{H}_{2} \mathrm{CO}$, and $\mathrm{C}_{3} \mathrm{H}_{2}$ toward positions in $\mathrm{B} 68$ offset from the extinction peak. We describe the BIMA observations and single-dish data in $\S 2$, and our method of determining abundances with a Monte Carlo radiative transfer code in $\S 3$. The derived results are discussed in $\S 4$. A concluding summary is found in $\S 5$.

\section{Observations and Results}

\subsection{BIMA Observations}

Figure 1 shows the position of the one pointing made toward B68 with the BIMA millimeter interferometer at Hat Creek, CA, i.e., at R.A./decl. $(2000)=17^{h} 22^{m} 38.6^{s}$, $-23^{\circ} 49^{\prime} 42.0^{\prime \prime}$, approximately $4^{\prime \prime}$ north of the extinction peak. Both ${ }^{13} \mathrm{CO} 1-0$ and $\mathrm{C}^{18} \mathrm{O} 1-0$

were observed simultaneously during tracks on 2001 May 09 and May 20, and $\mathrm{N}_{2} \mathrm{H}^{+} 1-0$, 
$\mathrm{HCO}^{+} 1-0$, and $\mathrm{HOC}^{+} 1-0$ were observed simultaneously on 2001 April 24 and May 14 . Figure 1 also shows the minimum and maximum FWHM sizes of the BIMA primary beams at the frequencies of ${ }^{13} \mathrm{CO} 1-0$ and $\mathrm{HCO}^{+} 1-0$. Each line was observed in its own correlator window of $6.25 \mathrm{MHz}$ width, with each window set to have 512 channels of $12.2 \mathrm{kHz}$ width (i.e., $\sim 0.03 \mathrm{~km} \mathrm{~s}^{-1}$ at $110 \mathrm{GHz}$.) The correlators were tuned so the central channel of each window corresponded to a $V_{L S R}$ of $+3.4 \mathrm{~km} \mathrm{~s}^{-1}$, the velocity centroid of $\mathrm{C}^{18} \mathrm{O} 2-1$ found by Wang et al. (1995).

Each track was only 4-5 hours in duration given the low maximum elevation of B68 from Hat Creek (i.e., $\sim 25^{\circ}$ ). Visibility phases were calibrated by observing 1733-130 for 5 minutes approximately every 30 minutes over $800 \mathrm{MHz}$ bandwidth in the lower or upper sideband. Visibility amplitudes were calibrated by similarly observing Uranus for 8 minutes at the end of each track. All data were reduced using standard routines within the MIRIAD software package (Sault, Teuben, \& Wright 1995). All channels were cleaned to $2 \sigma \mathrm{rms}$ intensity levels.

Line emission was not detected at any of the frequencies observed, at any positions toward B68 within the $\sim 2^{\prime}$ FWHMs of the BIMA primary beams. Binning together several channels or tapering the data with variously-sized Gaussians did not change this result. Table 1 summarizes these data, listing the lines, the source observatory, the synthesized beam FWHMs, and the angular offset of the BIMA pointing from the extinction peak. The $1 \sigma \mathrm{rms}$ sensitivities attained per channel ranged from $1.0 \mathrm{~K}$ for $\mathrm{HOC}^{+} 1-0$ to $1.5 \mathrm{~K}$ for ${ }^{13} \mathrm{CO} 1-0$, and were measured by sampling all channels within a $40^{\prime \prime} \times 40^{\prime \prime}$ box centered at the pointing center. Upper limits (at $3 \sigma$ ) to the integrated intensities of these lines ranged from $0.21 \mathrm{Jy}$ beam $^{-1} \mathrm{~km} \mathrm{~s}^{-1}$ to $0.34 \mathrm{Jy}_{\text {beam }}{ }^{-1} \mathrm{~km} \mathrm{~s}^{-1}$ (or $0.25 \mathrm{~K} \mathrm{~km} \mathrm{~s}^{-1}$ to $0.44 \mathrm{~K} \mathrm{~km} \mathrm{~s}^{-1}$ ) assuming a line width equal to $0.4 \mathrm{~km} \mathrm{~s}^{-1}$, the typical FWHM of optically-thin lines in B68 found by Wang et al. 


\subsection{Single-dish Observations}

We surveyed the literature to find previous detections of molecular line emission toward B68 from which abundances could be measured. Fortunately, B68 was included in numerous surveys of dark globules for molecular line emission. Early detections of lines include those of ${ }^{12} \mathrm{CO} 1-0$ and ${ }^{13} \mathrm{CO} 1-0$ by Martin \& Barrett (1978) and Leung, Kutner, \& Mead (1982), ${ }^{12}$ CO 2-1 by Avery et al. (1987) and Clemens, Yun, \& Heyer (1991), and ${ }^{12} \mathrm{CO} 3-2$ by Avery et al. Furthermore, $\mathrm{NH}_{3}(1,1)$ and $(2,2)$ were detected by Martin \& Barrett and Bourke et al. (1995). Although these data certainly associate line emission with B68, we exclude them from analysis because of their relatively low angular resolution, i.e., $>60^{\prime \prime}$ FWHM.

More recent detections of line emission from various species toward B68 have been made with resolutions $<60^{\prime \prime}$ FWHM, including those of $\mathrm{C}^{18} \mathrm{O} 2-1$ and $\mathrm{H}_{2} \mathrm{CO} 3_{12}-2_{11}$ by Wang et al., CS $2-1$ by Launhardt et al. (1998), $\mathrm{N}_{2} \mathrm{H}^{+} 1-0$ and $\mathrm{C}_{3} \mathrm{H}_{2} 2_{02}-1_{01}$ by Benson, Caselli, \& Myers (1998), and $\mathrm{NH}_{3}(1,1)$ by Lemme et al. (1996). Other recent detections

include those of $\mathrm{C}^{18} \mathrm{O} 1-0$ and $\mathrm{N}_{2} \mathrm{H}^{+} 1-0$ by Bergin et al., and ${ }^{13} \mathrm{CO} 1-0, \mathrm{C}^{18} \mathrm{O} 1-0$ and $2-1$ by Hotzel et al., but we exclude those data from our analysis because abundances have been specifically determined from them by the respective authors. An interesting non-detection in the literature is that of SO $1_{0}-0_{1}$ by Codella \& Muders (1997), but their map was centered on the nearby IRAS point source and did not overlap the region of extinction modeled by ALL01. The methods of observation and reduction relevant to these data are described in these references, and are not reproduced here.

In the higher-resolution single-dish studies, the positions where line characteristics were specifically reported vary widely. Figure 1 shows the positions and resolutions of observations from these studies against the $R$-band image of B68. Table 1 also lists the lines observed, the source observatories, the resolutions attained, and the angular offsets 
of the single-dish data from the extinction peak of the reported line characteristics. The positions listed in Table 1 for $\mathrm{C}^{18} \mathrm{O} 2-1$, CS 2-1, and $\mathrm{N}_{2} \mathrm{H}^{+} 1-0$ are those of peak line intensity from their respective maps, where line characteristics were specifically reported. $\left(\mathrm{H}_{2} \mathrm{CO} 3_{12}-2_{11}\right.$ and $\mathrm{C}_{3} \mathrm{H}_{2} 2_{02}-1_{01}$ were observed at only these positions by the respective authors.) Interestingly, these positions are not coincident with the extinction peak. The $\mathrm{C}^{18} \mathrm{O}$ and $\mathrm{CS}$ positions are approximately coincident with an arc of maximal $\mathrm{C}^{18} \mathrm{O} 1-0$ integrated intensity centered at the extinction peak noted by Bergin et al. (see their Figure 1a), and the $\mathrm{N}_{2} \mathrm{H}^{+}$position is approximately coincident with the "arc" of maximal $\mathrm{N}_{2} \mathrm{H}^{+}$ 1-0 integrated intensity about the extinction peak also noted by Bergin et al. (see their Figure 1b. $)^{5}$

\section{Monte-Carlo Radiative Transfer Models}

Molecular line data can place strong limits on molecular abundances within B68 because its density structure has been so well constrained by ALL01. For example, abundances can be estimated by calculating with a Monte Carlo code the radiative transfer, molecular excitation, and line emission through a model of a dense core with a specific density profile, and varying abundances within the model until the output matches the observed data. For this purpose, we used the one-dimensional Monte Carlo code of Hogerheijde \& van der Tak (2000) which can solve molecular excitation coupled with line and continuum radiative transfer in core models. This code is especially useful for application to B68. First, model fluxes can be found at positions arbitrarily offset from the central line-of-sight, to simulate

\footnotetext{
${ }^{5} \mathrm{An} \mathrm{N}_{2} \mathrm{H}^{+}$1-0 map toward B68 recently made by Caselli et al. (2002), confirms this position is that of peak intensity, although their data are not Nyquist sampled and are of lower resolution than those of Benson et al. and Bergin et al.
} 
observations of B68 at positions offset from the extinction peak. Second, the model fluxes can be sampled with the spatial frequency coverage of actual interferometer data to estimate how much emission may have been resolved out in those cases. Recently, van Zadelhoff et al. (2002) compared 8 molecular excitation/radiative transfer codes including that of Hogerheijde \& van der Tak, and found that all codes give results to within $10 \%$ of one another, even at high opacity.

For models of B68, we assumed the thermal and density structure given by ALL01, i.e, an isothermal sphere with an outer radius of 12500 AU and a "Bonnor-Ebert parameter" $\xi_{\max }$ of 6.9 , corresponding to a center-to-edge density ratio of 16.5 . Following ALL01, the temperature assumed in our models was $16 \mathrm{~K}$, as derived by Bourke et al. from $\mathrm{NH}_{3}$ observations of B68. (An alternative temperature profile is considered in $§ 4.1$ below.) The $\xi_{\max }$ parameter identifies the particular solution from the family that solves the second-order differential equation characterizing a Bonnor-Ebert sphere. We determined the density in 30 linearly-spaced concentric shells by solving that differential equation with a fourth-order Runge-Kutta method (see Press et al. 1992.) The central density in the ALL01 model for B68 is $\sim 2.5 \times 10^{5} \mathrm{~cm}^{-3}$.

Molecular abundances were assumed to be constant with radius, although abundance gradients within B68 are quite possible for the molecular species considered here. For example, Bergin et al. found edge-to-center contrasts in the abundances of $\mathrm{C}^{18} \mathrm{O}$ and $\mathrm{N}_{2} \mathrm{H}^{+}$ of 100 and 2 respectively using their own maps of B68. With only single pointings for the single-dish data and non-detections for the interferometer data, we can derive abundance values or upper limits only at the positions listed in Table 1, averaged over the respective lines-of-sight and beam widths, and cannot probe for abundance gradients. Gradients should not produce dramatic differences in the abundances we derive, however, given the small sizes of the beam widths relative to the spatial extent of B68. For example, we note 
in $\S 4.2$ little difference between our abundances of $\mathrm{C}^{18} \mathrm{O}$ and $\mathrm{N}_{2} \mathrm{H}^{+}$and those derived by Bergin et al. at positions observed in common, despite our different abundance profiles.

The velocity field of the gas was assumed to have a turbulent line width of $0.4 \mathrm{~km} \mathrm{~s}^{-1}$ (see Wang et al.; since the lines are optically thin at the abundances found, the integrated intensity is independent of the adopted turbulent line width.) We assume that each shell is stationary, although Bergin et al. suggest velocity variations within B68 may be significant. For reasons similar to those cited above for abundance gradients, we did not consider radial variations of line width.

In each shell, level populations were determined assuming standard collision rates for each molecule. The expected sky brightness distributions were then calculated using a $256 \times 256 \times 100$ cube with $1^{\prime \prime} \times 1^{\prime \prime} \times 0.04 \mathrm{~km} \mathrm{~s}^{-1}$ elements. To compare models with interferometer data, "visibility" datasets were produced from the cubes using the MIRIAD task uvmodel and the antenna baselines from the original data. These latter

datasets were inverted, cleaned and restored in the same manner as the actual data, and velocity-integrated line intensities calculated. By properly accounting for spatial filtering in this manner, we estimated BIMA would have recovered only $\sim 20 \%$ of the flux emitted from an ALL01 Bonnor-Ebert sphere in B68. To compare models with single-dish data, the original cubes were convolved with Gaussians with widths appropriately representing the resolution of the respective observations. The abundances were varied until the observational data were well matched.

\section{Abundances}

Table 2 lists the fractional abundances of the sampled molecular species at various positions toward B68, constrained with the data described in $\S 2$, and estimated by using 
the method described in $\S 3$ (assuming models with the Bonnor-Ebert density profile of ALL01.) The BIMA data yield the first upper limits to the abundances of $\mathrm{HCO}^{+}$toward the extinction peak of $\mathrm{B} 68$, as well as upper limits to the abundances of ${ }^{13} \mathrm{CO}, \mathrm{C}^{18} \mathrm{O}$ and $\mathrm{N}_{2} \mathrm{H}^{+}$at the same location. Since collision rates are not available for $\mathrm{HOC}^{+}$, we do not attempt to place limits on its abundance. In addition, single-dish data of B68 from the literature yield abundance values of $\mathrm{H}_{2} \mathrm{CO}, \mathrm{CS}, \mathrm{NH}_{3}$, and $\mathrm{C}_{3} \mathrm{H}_{2}$ toward $\mathrm{B} 68$ for the first time, but at various positions offset from the extinction peak. Finally, other single-dish data of $\mathrm{B} 68$ from the literature yield new abundance values of $\mathrm{C}^{18} \mathrm{O}$ and $\mathrm{N}_{2} \mathrm{H}^{+}$at some of these latter locations.

\subsection{Comparison with Other Clouds}

How do the abundances derived for B68 here compare to those derived for other clouds? "Standard" cloud molecular abundances with which to base comparisons are difficult to define given wide variations in chemical environment or evolutionary epoch in the ISM. To provide some comparison, Table 2 also lists the fractional abundance estimates of the same species in clouds similar in character to B68, obtained directly from the literature but made without the detailed foreknowledge of cloud density structure now available for B68. The first set, for "translucent clouds," are those listed by Turner (2000) for small, round Clemens \& Barvainis clouds with edge-to-center visual extinctions of 2.0. These clouds were modeled with constant abundance, and the listed values are averages between the results of a hydrostatic equilibrium polytropic model and an $n(r) \propto r^{0} \operatorname{model}^{6}$. The second set, denoted for "cold dense clouds," are those compiled by Ohishi, Irvine, \& Kaifu (1992) for

\footnotetext{
${ }^{6}$ According to Turner (2002, private communication), these two models produce results that differ at most by a factor of 1.35 for species that are highly dependent on density.
} 
a single position in TMC-1 or L134N, and were made using column density estimates from many authors, assuming $\mathrm{N}\left(\mathrm{H}_{2}\right)=10^{22} \mathrm{~cm}^{-2}$ (see Turner, or Pratap et al. 1997, or Dickens et al. 2000 for alternative estimates.) The abundances from Turner and Ohishi et al. in Table 2 should not be regarded as universal. For example, Turner notes abundances can vary by an order of magnitude both between different translucent clouds and within larger, dense clouds (presumably determined using similar assumptions.)

Table 2 shows that every value of molecular abundance we derive for B68 is less than the lowest value derived for other clouds. From the BIMA data, we find the upper limits to the ${ }^{13} \mathrm{CO}, \mathrm{HCO}^{+}$, and $\mathrm{N}_{2} \mathrm{H}^{+}$abundances in $\mathrm{B} 68$ are lower than values derived for other clouds by factors of 7,14 , and 4 respectively. (The upper limit to the $\mathrm{C}^{18} \mathrm{O}$ abundance is similar to the lowest value found for other clouds.) From the various single-dish data, we find the $\mathrm{C}^{18} \mathrm{O}, \mathrm{CS}$, and $\mathrm{H}_{2} \mathrm{CO}$ abundances differ least, by roughly an order of magnitude or less, with the B68 abundances lower than the lowest values derived for other clouds by factors of only 5, 3, and 16 respectively at the locations observed. However, the $\mathrm{N}_{2} \mathrm{H}^{+}$, $\mathrm{NH}_{3}$, and $\mathrm{C}_{3} \mathrm{H}_{2}$ abundances differ the most, by over an order of magnitude, with those of B68 lower by factors of 25, 29, and 170 respectively at the locations observed.

The assumption in our models of an isothermal Bonnor-Ebert sphere, at the same $16 \mathrm{~K}$ temperature derived by Bourke et al. and used by ALL01, has likely minimized the resulting abundance values we obtain. Other temperature profiles, such as those suggested for Bonnor-Ebert spheres by Zucconi, Walmsley, and Galli (2001; see also Evans et al. 2001) which have cooler temperatures at small radii, may be more appropriate. For example, Bergin et al. assumed for their models of B68 the Zucconi et al. radial temperature profile with a global reduction of $2 \mathrm{~K}$, in addition to the ALL01 density profile. With this same temperature profile in our models, the values in Table 2 increase by factors of only $2-3$. Therefore, using this radial temperature profile would bring the abundances of $\mathrm{C}^{18} \mathrm{O}$, CS, 
and $\mathrm{H}_{2} \mathrm{CO}$ at the positions observed in $\mathrm{B} 68$ even more in line with the abundances found for other cloud types. However, the abundances of $\mathrm{N}_{2} \mathrm{H}^{+}, \mathrm{NH}_{3}$, and $\mathrm{C}_{3} \mathrm{H}_{2}$ at other positions would still remain lower than those of other clouds by an order of magnitude or more.

Only $\mathrm{N}_{2} \mathrm{H}^{+}, \mathrm{NH}_{3}$, and $\mathrm{C}_{3} \mathrm{H}_{2}$ are arguably depleted in $\mathrm{B} 68$, given the values shown in Table 2 and assuming a typical order of magnitude abundance variation between and within similar clouds. However, other species considered here still may be depleted in B68. Note that the degree to which the abundance of a particular species in B68 is low compared to those of other clouds appears related to the position across B68 that the respective data were obtained. The $\mathrm{C}^{18} \mathrm{O}, \mathrm{CS}$, and $\mathrm{N}_{2} \mathrm{H}^{+}$data from the literature were those of maximum line brightness from maps of $\mathrm{B} 68$, and $\mathrm{H}_{2} \mathrm{CO}$ and $\mathrm{C}_{3} \mathrm{H}_{2}$ data were obtained only subsequently by the respective authors at those positions. $\left(\mathrm{NH}_{3}\right.$ was observed only at the Clemens \& Barvainis position of B68.) Figure 1 and Table 2 together reveal that the less-discrepant abundances are found at positions relatively far from the extinction peak but the more-discrepant abundances are found at positions closer to the extinction peak (except notably $\mathrm{NH}_{3}$.) This pattern suggests $\mathrm{C}_{3} \mathrm{H}_{2}$ may be also depleted by some process related to extinction, e.g., the sublimation of gas-phase molecules onto grains, as suggested by Bergin et al. for $\mathrm{C}^{18} \mathrm{O}$ and $\mathrm{N}_{2} \mathrm{H}^{+}$and Hotzel et al. for ${ }^{13} \mathrm{CO}$ and $\mathrm{C}^{18} \mathrm{O}$. This same idea may also

explain how $\mathrm{CS}$ and $\mathrm{H}_{2} \mathrm{CO}$ appear relatively undepleted in the outer, less-extincted radii of B68. Moreover, the lack of bright emission in CS near the extinction peak hint it may be also depleted at high extinction in B68. These speculations can be confirmed only after analyzing fully-sampled, high-resolution line maps of B68.

\subsection{Comparison with Previous Abundance Determinations}

How do the abundances we derive compare with those found previously for B68? Bergin et al. found the $\mathrm{C}^{18} \mathrm{O}$ abundance in $\mathrm{B} 68$ rises from very low values at $A_{V}<<1$ 
to a peak of $1 \times 10^{-7}$ at $A_{V}=2$, and decreases to $1 \times 10^{-9}$ at $A_{V}>20$, a contrast of 100. (Hotzel et al. obtained edge-to-center $\mathrm{C}^{18} \mathrm{O}$ abundance contrasts of 2-200 in their models.) In addition, Bergin et al. found the $\mathrm{N}_{2} \mathrm{H}^{+}$abundance rises from very low values at $A_{V}<<1$ to a peak of $6 \times 10^{-11}$ at $A_{V}=3$, and decreases to $3 \times 10^{-11}$ at $A_{V}>20$, a contrast of $\sim 2$. Any evidence of $\mathrm{N}_{2} \mathrm{H}^{+}$depletion is remarkable, given its oft-described utility as a non-depleting probe of dense core interiors (e.g., see Tafalla et al. 2002.)

Our interferometer data of $\mathrm{C}^{18} \mathrm{O}$ and $\mathrm{N}_{2} \mathrm{H}^{+}$do not provide much additional support for the abundance model for B68 of Bergin et al. Our derived upper limits for $\mathrm{C}^{18} \mathrm{O}$ and $\mathrm{N}_{2} \mathrm{H}^{+}$from the BIMA data are not particularly low, but they remain consistent with the still-lower values found by Bergin et al. throughout the core. Also, the non-detection of compact line emission with BIMA suggests the low abundances found by Bergin et al. are not due to the single-dish beam dilution of small clumps of relatively abundant material. A spatially-smoother model of gas density is indeed more appropriate for B68, such as the Bonnor-Ebert sphere suggested by the dust extinction maps.

The single-dish $\mathrm{C}^{18} \mathrm{O}$ and $\mathrm{N}_{2} \mathrm{H}^{+}$data from the literature only pertain to one line-of-sight per transition toward B68, but provide strong support for the abundances derived by Bergin et al., if the same modified Zucconi et al. temperature profile is assumed. With this assumption, the $\mathrm{C}^{18} \mathrm{O}$ literature data, from a position $599^{\prime \prime} 4$ offset from the extinction peak, yield an apparent abundance of $6-9 \times 10^{-8}$, quite consistent with the $3-9 \times 10^{-8}$ abundance range expected along this line-of-sight from the model of Bergin et al. Also, the $\mathrm{N}_{2} \mathrm{H}^{+}$literature data, from a position $16^{\prime \prime} .3$ offset from the extinction peak, yield an apparent value of $4-6 \times 10^{-11}$, slightly larger than but still consistent with the $3-4 \times$ $10^{-11}$ abundance range expected along this line-of-sight from the model of Bergin et al. Furthermore, the observed integrated intensities of these literature data at each respective location and our BIMA observations can be reproduced to within only a few percent with 
our own Monte Carlo models, assuming the ALL01 density profile, the modified Zucconi et al. temperature profile and the Bergin et al. radial abundance profiles. (Note that here we relaxed the earlier assumption of constant abundance with radius.)

\section{Summary and Conclusions}

Using our own interferometer line data and single-dish line data culled from the literature of the Bok globule B68 and a Monte-Carlo radiative transfer code, we derive abundances for $\mathrm{C}^{18} \mathrm{O}, \mathrm{CS}, \mathrm{N}_{2} \mathrm{H}^{+}, \mathrm{NH}_{3}, \mathrm{H}_{2} \mathrm{CO}$, and $\mathrm{C}_{3} \mathrm{H}_{2}$ and upper limits for abundances of ${ }^{13} \mathrm{CO}$ and $\mathrm{HCO}^{+}$. Foreknowledge of the Bonnor-Ebert density configuration of B68, as found from extinction mapping by ALL01, has removed a major uncertainty that affected previous estimations of abundances in other clouds. We find molecular abundances in B68 are lower than those estimated previously for clouds similar to $\mathrm{B} 68 . \mathrm{N}_{2} \mathrm{H}^{+}, \mathrm{NH}_{3}$, and $\mathrm{C}_{3} \mathrm{H}_{2}$ have abundances lower than the lowest values determined for other clouds by 1.4 , 1.5 , and 2.2 orders of magnitude respectively, but abundances of the other species are only lower by one order of magnitude or less. Depletion of CS is suggested by the large offset between the position of maximum CS line brightness and the extinction peak. Furthermore, abundances of $\mathrm{C}^{18} \mathrm{O}$ and $\mathrm{N}_{2} \mathrm{H}^{+}$derived using data from the literature at locations where line characteristics were reported are consistent with those derived by Bergin et al.

As shown by Bergin et al., fully-sampled maps from single-dish telescopes can more effectively determine abundances within B68. Further maps of B68 can be now easily obtained using the new multi-beam focal-plane arrays on current single-dish telescopes. Furthermore, the upcoming generation of millimeter interferometers will have the sensitivity to provide additional data for combined maps of even higher resolution. With these data, more accurate molecular abundances will be determined for B68. For example, it will be interesting to determine if abundances of $\mathrm{CS}, \mathrm{HCO}^{+}, \mathrm{NH}_{3}, \mathrm{H}_{2} \mathrm{CO}$, and $\mathrm{C}_{3} \mathrm{H}_{2}$ also vary 
with extinction, like ${ }^{13} \mathrm{CO}, \mathrm{C}^{18} \mathrm{O}$, or $\mathrm{N}_{2} \mathrm{H}^{+}$. Similar abundance determinations across other isolated dense cores may also be possible, provided their density structures can be similarly well-defined.

We thank Barry Turner, our referee, for insightful comments that improved this paper. In addition, we thank Leo Blitz for allowing this project to be pursued with the BIMA millimeter array. JD's research in Berkeley was supported by the Radio Astronomy Laboratory. MRH's research in Berkeley was supported by the Miller Institute for Basic Research in Science. We also thank Charles J. Lada, João Alves, Tracy Huard, and Jon Swift for their help. This research has made use of the SIMBAD database and Aladin, both operated by CDS, Strasbourg, France. 


\section{REFERENCES}

Alves, J. F., Lada, C. J., \& Lada, E. A. 2001, Nature, 409, 159 (ALL01)

Avery, L. W., White, G. J., Williams, I. P., \& Cronin, N. 1987, ApJ, 312, 848

Barnard, E. E. 1919, ApJ, 49, 1

Benson, P. J., Caselli, P, \& Myers, P. C. 1998, ApJ, 506, 743

Bergin, E. A., Alves, J., Huard, T., \& Lada, C. J. 2002, ApJ, 570, L101

Bok, B., \& Reilly, E. F. 1947, ApJ, 105, 255

Bourke, T. L., Hyland, A. R., Robinson, G., James, S. D., \& Wright, C. M., 1995, MNRAS, 276,1067

Bonnor, W. B. 1956, MNRAS, 116, 351

Caselli, P., Benson, P. J., Myers, P. C., \& Tafalla, M. 2002, ApJ, in press

Clemens, D. P., \& Barvainis, R. 1988, ApJS, 68, 257

Clemens, D. P., Yun, J. L., \& Heyer, M. H. 1991, ApJS, 75, 877

Codella, C., \& Muders, D. 1997, MNRAS, 291, 337

de Geus, E. J., de Zeeuw, P. T., \& Lub, J. 1989, A\&A, 216, 44

Dickens, J. E., Irvine, W. M., Snell, R. L., Bergin, E. A., Schloerb, F. P., Pratap, P., \& Miralles, M. P. 2000, ApJ, 542, 870

Ebert, R. 1955, Z. Astrophys., 37, 217

Evans, N. J., II, Rawlings, J. M. C., Shirley, Y. L., \& Mundy, L. G. 2001, ApJ, 557, 193

Hogerheijde, M. R. \& van der Tak, F. F. S. 2000, A\&A, 362, 697

Hotzel, S., Harju, J., Juvela, M., Mattila, K., \& Haikala, L. K. 2002, A\&A, in press 
Launhardt, R., Evans, N. J., II, Wang, Y., Clemens, D. P., Henning, Th., \& Yun, J. L. 1998, ApJS, 119, 59

Launhardt, R., \& Henning, Th. 1997, A\&A, 326, 329

Lemme, C., Wilson, T. L., Tieftrunk, A. R., \& Henkel, C. 1996, A\&A, 312, 585

Leung, C. M., Kutner, M. L., \& Mead, K. N. 1982, ApJ, 262, 583

Martin, R. N., \& Barrett, A. H. 1978, ApJS, 36, 1

Ohishi, M., Irvine, W. M., \& Kaifu, N. 1992, in IAU Symposium 150, Astrochemistry of Cosmic Phenomena: Proceedings of the 150th Symposium of the International Astronomical Union, ed. P. D. Singh (Dordrecht: Reidel), 171

Parker, N. D. 1988, MNRAS, 235, 139

Pratap, P., Dickens, J. E., Snell, R. L., Miralles, M. P., Bergin, E. A., Irvine, W. M., \& Schloerb, F. P. 1997, ApJ, 486, 862

Press, W. H., Teukolsky, S. A., Vetterling, W. T., \& Flannery, B. P. 1992, Numerical Recipes (Cambridge: Cambridge University Press)

Reipurth, B., Nyman, L.-A, \& Chini, R. 1996, A\&A, 315, 258

Sault, R. J., Teuben, P. J., \& Wright, M. C. H. 1995, in ASP Conf. Ser. 77, Astronomical Data Analysis Software and Systems IV, ed. R. A. Shaw, H. E. Payne, \& J. J. E. Hayes (San Francisco: Astronomical Society of the Pacific), 433

Tafalla, M., Myers, P. C., Caselli, P., Walmsley, C. M., \& Comito, C. 2002, ApJ, 569, 815

Turner, B. E. 2000, ApJ, 542, 837

van Zadelhoff, G.-J., Dullemond, C. P., van der Tak, F. F. S., Yates, J. A., Doty, S. D., Ossenkopf, V., Hogerheijde, M. R., Juvela, M., Wiesemeyer, H., \& Schöier, F. L. 2002, A\&A, submitted 
Wang, Y., Evans, N. J., II, Zhou, S., \& Clemens, D. P. 1995, ApJ, 454, 217

Zucconi, A., Walmsley, C. M., \& Galli, D. 2001, A\&A, 376, 650 
Fig. 1.- ESO MAMA $R$-band image of B68 (from CDS/Aladin). The position of maximum visual extinction determined by ALL01, i.e., the extinction peak, is denoted by a diamond. The central pointing position of the BIMA observations is shown as a cross and the FWHM of the BIMA primary beams at $89.2 \mathrm{GHz}\left(\mathrm{HCO}^{+} 1-0\right)$ and $110.2 \mathrm{GHz}\left({ }^{13} \mathrm{CO} 1-0\right)$ are shown respectively as the outer and inner dashed circles. The positions and FWHM beam sizes of single-dish line observations from the literature are shown respectively as triangles and dotted circles. From north to south, these positions are from Launhardt et al. (1998; CS 2-1), Benson et al. (1998; $\mathrm{N}_{2} \mathrm{H}^{+} 1-0$ and $\mathrm{C}_{3} \mathrm{H}_{2} 2_{12}-1_{01}$ ), Wang et al. (1995; $\mathrm{C}^{18} \mathrm{O} 2-1$ and $\left.\mathrm{H}_{2} \mathrm{CO} 3_{21}-2_{11}\right)$, and Lemme et al. (1996; $\left.\mathrm{NH}_{3}(1,1)\right)$. The "X" denotes the position of $\mathrm{B} 68$ from Clemens \& Barvainis (1988). The error ellipse of the nearby far-infrared point source IRAS 17194-2351 is located south of B68 off the side of the image shown. The scale bar size assumes a distance to $\mathrm{B} 68$ of 125 pc. 
Table 1. Summary of Line Observations of B68

\begin{tabular}{|c|c|c|c|c|}
\hline Line & Observatory & $\begin{array}{l}\text { Beam FWHM } \\
\qquad\left({ }^{\prime \prime} \times{ }^{\prime \prime}\right)\end{array}$ & $\begin{array}{l}\text { Extinction Peak Offset } \\
\left.\text { (" }{ }^{\text {b }}\right)\end{array}$ & Reference \\
\hline${ }^{13} \mathrm{CO} 1-0$ & BIMA & $17.1 \times 4.7$ & 4.03 & 1 \\
\hline $\mathrm{C}^{18} \mathrm{O} 1-0$ & BIMA & $17.7 \times 4.5$ & 4.03 & 1 \\
\hline $\mathrm{C}^{18} \mathrm{O} 2-1$ & $\mathrm{CSO}$ & $30 \times 30$ & $59.3^{\mathrm{c}}$ & 2 \\
\hline CS 2-1 & FCRAO & $46 \times 46$ & $66.2^{\mathrm{c}}$ & 3 \\
\hline $\mathrm{HCO}^{+} 1-0$ & BIMA & $18.5 \times 7.0$ & 4.03 & 1 \\
\hline $\mathrm{HOC}^{+} 1-0$ & BIMA & $18.9 \times 7.0$ & 4.03 & 1 \\
\hline $\mathrm{N}_{2} \mathrm{H}^{+} 1-0$ & BIMA & $17.7 \times 6.6$ & 4.03 & 1 \\
\hline $\mathrm{N}_{2} \mathrm{H}^{+} 1-0$ & Haystack & $18 \times 18$ & 16.3 & 4 \\
\hline $\mathrm{NH}_{3}(1,1)$ & Effelsberg & $32 \times 32$ & 54.5 & 5 \\
\hline $\mathrm{H}_{2} \mathrm{CO} 3_{12}-2_{11}$ & $\mathrm{CSO}$ & $30 \times 30$ & 59.3 & 2 \\
\hline $\mathrm{C}_{3} \mathrm{H}_{2} 2_{12}-1_{01}$ & Haystack & $18 \times 18$ & 16.3 & 4 \\
\hline
\end{tabular}

References - (1) this work, (2) Wang et al. 1995, (3) Launhardt et al. 1998, (4) Benson, Caselli \& Myers 1998, (5) Lemme et al. 1996.

aSynthesized beam FWHM for BIMA data, observed beam FWHM for single-dish data reported by authors.

${ }^{\mathrm{b}}$ Offset of data from the extinction peak.

${ }^{c}$ Position of peak emission from map, where line characteristics were reported by authors. 
Table 2. Abundances of Various Molecular Clouds

\begin{tabular}{ccccc}
\hline \hline Species & B68 $^{\mathrm{a}}$ & $\begin{array}{c}\text { Translucent } \\
\text { Clouds }^{\mathrm{b}}\end{array}$ & TMC-1 $^{\mathrm{c}}$ & L134N $^{\mathrm{c}}$ \\
\hline${ }^{13} \mathrm{CO}$ & $<1.3(-7)$ & $\ldots$ & $8.9(-7)^{\mathrm{d}}$ & $8.9(-7)^{\mathrm{d}}$ \\
$\mathrm{C}^{18} \mathrm{O}$ & $<1.3(-7)$ & $\ldots$ & $1.6(-7)^{\mathrm{d}}$ & $1.6(-7)^{\mathrm{d}}$ \\
$\mathrm{C}^{18} \mathrm{O}$ & $3.0(-8)$ & $\ldots$ & $1.6(-7)$ & $1.6(-7)$ \\
$\mathrm{CS}^{2}-1$ & $4.0(-10)$ & $1.1(-9)$ & $1(-8)$ & $1(-9)$ \\
$\mathrm{HCO}^{+}$ & $<1.4(-10)$ & $2(-9)$ & $8(-9)$ & $8(-9)$ \\
$\mathrm{HOC}^{+}$ & $\cdots$ & $\ldots$ & $\cdots$ & $\cdots$ \\
$\mathrm{N}_{2} \mathrm{H}^{+}$ & $<1.3(-10)$ & $1(-9)$ & $5(-10)$ & $5(-10)$ \\
$\mathrm{N}_{2} \mathrm{H}^{+}$ & $2.0(-11)$ & $1(-9)$ & $5(-10)$ & $5(-10)$ \\
$\mathrm{NH}_{3}$ & $7.0(-10)$ & $2.1(-8)$ & $2(-8)$ & $2(-7)$ \\
$\mathrm{H}_{2} \mathrm{CO}$ & $4.0(-10)$ & $6.3(-9)$ & $2(-8)$ & $2(-8)$ \\
$\mathrm{C}_{3} \mathrm{H}_{2}$ & $1.2(-11)$ & $3.6(-8)$ & $3(-8)$ & $2(-9)$ \\
\hline
\end{tabular}

${ }^{a}$ Abundances derived at positions listed in Table 1 assuming Bonnor-Ebert model of ALL01 and T $=16 \mathrm{~K} . \mathrm{Up}-$ per limits are derived from interferometer non-detections while values are derived from single-dish detections described in the literature. Using the temperature profile suggested by Zucconi, Walmsley, \& Galli (2001) increases abundances by factors of $2-3$.

b Translucent cloud abundances from Turner (2000) are defined for a fiducial cloud of constant abundance with edge-to-center visual extinction of 2.0. The values listed are averages from hydrostatic equilibrium polytropic models and constant-density models.

${ }^{\mathrm{c}}$ Cold dense cloud abundances from Ohishi, Irvine, \& Kaifu (1992), assuming $\mathrm{N}\left(\mathrm{H}_{2}\right)=10^{22} \mathrm{~cm}^{-2}$.

${ }^{\mathrm{d}}$ Derived from ${ }^{12} \mathrm{CO}$ abundance assuming ${ }^{12} \mathrm{C} /{ }^{13} \mathrm{C}=90$ or ${ }^{16} \mathrm{O} /{ }^{18} \mathrm{O}=500$.

Note. $-a(-b)$ denotes $a \times 10^{-b}$ 


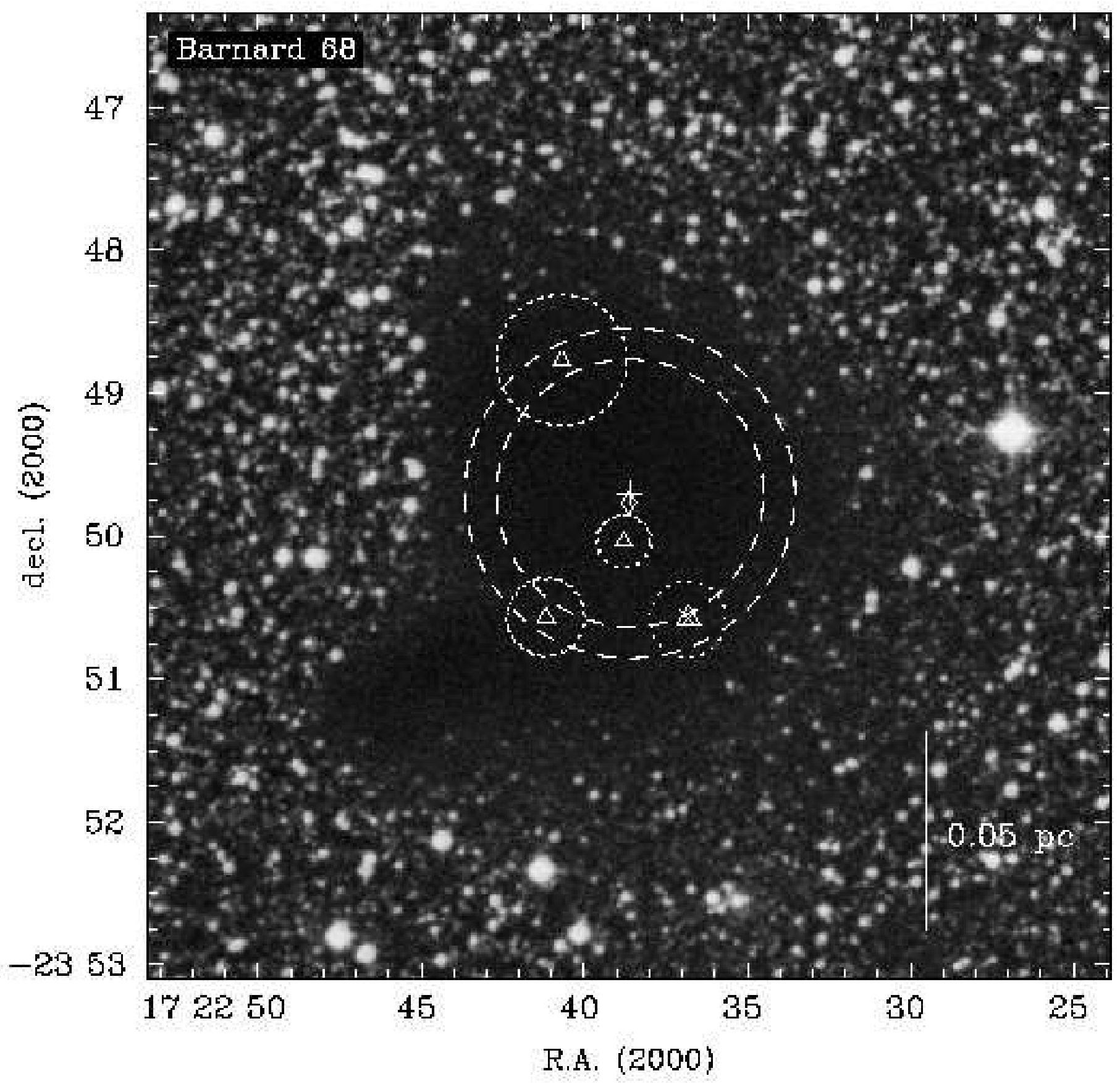

